\title{
ORTHOGONALITY AND THE HAUSDORFF DIMENSION OF THE MAXIMAL MEASURE
}

\author{
ARTUR OSCAR LOPES
}

\begin{abstract}
In this paper the orthogonality properties of iterated polynomials are shown to remain valid in some cases for rational maps. Using a functional equation fulfilled by the generating function, the author shows that the Hausdorff dimension of the maximal measure is a real analytical function of the coefficients of an Axiom A rational map satisfying the property that all poles of $f$ and zeros of $f^{\prime}(z)$ have multiplicity one.
\end{abstract}

Here we will consider $f$ a rational map such that the Julia set (see [1]) is bounded and $f$ is of the form $f(z)=P(z)(Q(z))^{-1}$, where $P(z)=z^{n}+a_{n-1} z^{n-1}$ $+\cdots+a_{1} z+a_{0}, Q(z)=b_{d} z^{d}+b_{d-1} z^{d-1}+\cdots+b_{1} z+b_{0}$, where $a_{i} \in \mathbb{C}, b_{j} \in$ $\mathbb{C}, b_{d} \neq 0, n>2$, and $d<n$.

In $[6,8$, and 10] it was shown that for $f$ a rational map there exists just one $f$-invariant probability measure $u$ such that, for any continuous function $\Phi$,

$$
\int \Phi(x) d u(x)=n^{-1} \int \sum_{i=1} \Phi\left(z_{i}(x)\right) d u(x)
$$

where $z_{i}(x), i \in\{1, \ldots, n\}$, are the roots of $f(z)=x$, counted with multiplicity, and this is the measure of maximum entropy. This measure is called the maximal measure, and it has entropy $\log n$. For $f$ such that $f(\infty)=\infty$ and $J(f)$ bounded, this measure is the equilibrium measure for the logarithm potential if and only if $f$ is a polynomial $[1,9]$.

Let $F(z)$ be the only one such that $F(z) / z$ is analytic near $\infty, F(z) \sim z$ as $z \rightarrow \infty$, and

$$
F^{\prime}(z) F(z)^{-1}=\int(z-x)^{-1} d u(x)=z^{-1}\left(\sum_{m=0}^{\infty} M_{m} z^{-m}\right),
$$

where $M_{m}=\int x^{m} d u(x)$ for $m \in N$ (see [2]) are the $m$-moments of $u$.

Note that $M_{0}=1$, and the expansion is valid only when the Julia set is bounded, which implies either $d<n-1$ or $d=n-1$, and $\left|b_{d}\right|<1$ or $\left|b_{d}\right|=1$, and there is a Siegel disk around infinity.

Received by the editors August 16, 1985.

Key words and phrases. Hausdorff dimension, rational maps, orthogonality, Axiom A, generating function.

1980 Mathematics Subject Classification. Primary 42A52, 58F11. 
We will consider $d=n-1$ in Theorems 1 and 2 just to simplify the formulas. The same result can be easily obtained in the same way in the general case $d<n$. In Theorems 3 and 4 , the interesting case is for $d=n-1$, and the formulas of Theorem 1 will be used there.

THEOREM 1. Let $s_{m}=\sum_{i=1}^{n} p_{i}^{m}$ and $t_{m}=\sum_{j=1}^{d} q_{j}^{m}$, where $d=n-1$ and $p_{i}$ and $q_{j}$ are respectively the zeros of $P$ and $Q$. Let $a_{k}^{m}$ be the coefficient of $z^{-k}$ in the Laurent series in $\infty$ of $f(z)^{-m}$ where $m, k \in \mathbb{N}$, then $M_{m}$ is obtained recursively by

$$
M_{m}=\left(n-a_{m}^{m}\right)^{-1}\left[s_{m}+\sum_{j=1}^{m-1} M_{j} \sum_{i=0}^{m-j} a_{m-i}^{j}\left(s_{i}-t_{i}\right)\right] \text {. }
$$

Proof. The following functional equation was obtained in [9]:

$$
f^{\prime}(z) \int(f(z)-x)^{-1} d u(x)=n \int(z-x)^{-1} d u(x)-\sum_{i=1}^{d}\left(z-q_{i}\right)^{-1} \text {. }
$$

To obtain the Laurent series in $\infty$ of

$$
f^{\prime}(z) \int(f(z)-x)^{-1} d u(x)=f^{\prime}(z) f(z)^{-1} \sum_{m=0}^{\infty} M_{m} f(z)^{-m},
$$

we have to obtain the Laurent series of $M_{m} f^{\prime}(z) f(z)^{-(m+1)}$. This series is obtained in the following way:

$$
\begin{aligned}
M_{m} f^{\prime}(z) f(z)^{-1} f(z)^{-m} & =M_{m}\left(P^{\prime}(z) P(z)^{-1}-Q^{\prime}(z) Q(z)^{-1}\right) f(z)^{-m} \\
& =M_{m} z^{-1}\left(\sum_{i=0}^{\infty}\left(s_{i}-t_{i}\right) z^{-i}\right)\left(\sum_{k=m}^{\infty} a_{k}^{m} z^{-k}\right) \\
& =M_{m} z^{-1} \sum_{j=0}^{\infty}\left(\sum_{i=0}^{j} a_{m+j-i}^{m}\left(s_{i}-t_{i}\right)\right) z^{-(m+j)}
\end{aligned}
$$

We point out that $a_{m}^{m}=\left(b_{d}\right)^{m}$ for $m \geqslant 0$, the first term in the above expression is $M_{m} b_{d}^{m}\left(s_{0}-t_{0}\right) z^{-(m+1)}$, and we have $\left(s_{0}-t_{0}\right)=n-d=1$.

The Laurent series in $\infty$ of $f^{\prime}(z) \int(f(z)-x)^{-1} d u(x)$ is

$$
\begin{aligned}
f^{\prime}(z) f(z)^{-1}\left(\sum_{m=0}^{\infty} M_{m} f(z)^{-m}\right) & \\
& =\sum_{m=0}^{\infty} M_{m} f^{\prime}(z) f(z)^{-1} f(z)^{-m} \\
& =z^{-1}\left(\sum_{v=0}^{\infty}\left[\left(\sum_{j=1}^{v} M_{j} \sum_{i=0}^{v-j} a_{v-i}^{j}\left(s_{i}-t_{i}\right)\right)+\left(s_{v}-t_{v}\right)\right] z^{-v}\right) .
\end{aligned}
$$

The Laurent development of

$$
n \int(z-x)^{-1} d u(x)-\sum_{i=1}^{d}\left(z-q_{i}\right)^{-1}
$$

is

$$
z^{-1}\left(\sum_{v=0}^{\infty}\left(n M_{v}-t_{v}\right) z^{-v}\right)
$$


Therefore

$$
n M_{m}-t_{m}=\left(s_{m}-t_{m}\right)+M_{m} a_{m}^{m}+\sum_{j=1}^{m-1} M_{j}\left(\sum_{i=0}^{m-j} a_{m-i}^{j}\left(s_{i}-t_{i}\right)\right) .
$$

Finally, $M_{m}$ can be obtained inductively by

$$
M_{m}=\left(n-a_{m}^{m}\right)^{-1}\left[s_{m}+\sum_{j=1}^{m-1} M_{j}\left(\sum_{i=0}^{m-j} a_{m-i}^{j}\left(s_{i}-t_{i}\right)\right)\right]
$$

Definition 1. $f$ is expanding if there exists a $k \in \mathbb{N}$ such that $\left|\left(f^{k}\right)^{\prime}(x)\right|>1$ for any $z$ in the Julia set.

Definition 2. The Hausdorff dimension of a measure $u$ is the inf $\{$ Hausdorff dimension of $\Lambda$ for all measurable sets such that $u(\Lambda)=1$ \}.

Ruelle [12] showed that the Hausdorff dimension of the Julia set of an expanding rational map is a real analytic function of the coefficients. Here we will show

THEOREM 2. Suppose $f_{\lambda}$ is a family of expanding rational maps with coefficients depending analytically on $\lambda \in \mathbb{R}$ such that $f_{\lambda}(z)$ has all poles and $f_{\lambda}^{\prime}(z)$ has all zeros with algebraic multiplicity one. Then Hausdorff dimension of the maximal measure of $f_{\lambda}$ is real analytic with respect to the parameter $\lambda$. If all zeros and all poles are respectively in the same component of $\mathbb{C}-J\left(f_{\lambda}\right)$, then the condition on the zeros and poles is unnecessary.

Proof. By [11] the Hausdorff dimension of $u$ satisfies

$$
\begin{aligned}
H D(u) & =\text { entropy of } u\left(\int \log \left|f^{\prime}(x)\right| d u(x)\right)^{-1} \\
& =\log n\left(\sum_{i=1}^{r} \int \log \left|x-r_{i}\right| d u(x)-\sum_{j=1}^{v} \int \log \left|x-v_{j}\right| d u(x)-\log b_{n-1}\right)^{-1},
\end{aligned}
$$

where $r_{i}$ and $v_{j}$ are resepctively the zeros and poles of $f^{\prime}$ counted with multiplicity.

Since $\log |F(z)|=\int \log |z-x| d u(x)$, we have

$$
H D(u)=\log d\left(\sum_{i=1}^{r} F\left(r_{i}\right)-\sum_{j=1}^{v} F\left(v_{j}\right)-\log b_{n-1}\right)^{-1} .
$$

We claim that the coefficients of the Laurent series of $F(z)$ depend analytically on the coefficients of $f(z)$. From [7, Theorem 17.3.2] the coefficients of $F(z)$ depend analytically on the moments $M_{m}$. Now, by (1), each moment $M_{m}$ is a finite sum of $s_{j}, t_{j}, a_{j}^{k}$, which are themselves analytic on the coefficients of $f(z)$. Therefore the claim is proved.

Now since the sum of the values of an analytic map in the roots of a polynomial is an analytic function of the coefficients of the polynomial, we conclude that the Hausdorff dimension of the maximal measure is a real analytic function of the coefficients of $f(x)$.

Consider the sequence $\left\{f^{n}(z)\right\}, n \in \mathbb{N}$, where $f^{0}(z)=z$ and $f^{n}(z)=f \circ f^{n-1}(z)$. In [2] conditions were given for the orthogonality of the sequences $\left\{f^{n}\right\}$ with respect to the measure $u$ when $f$ is a polynomial (that is, $\int f^{m}(z) f^{n}(z) d u(z)=0$ for $m \neq n)$. See also $[3,4$ and 5]. 
Here we are using a nonhermitian scalar product similar to the one used in [2].

EXAMPLE. For $f(z)=z^{n}$ the maximal measure is Lebesgue measure on the unit circle, and orthogonality is a consequence of the orthogonality of the Fourier series.

For $f$ a rational map such that $f(\infty)=\infty$, the interesting case is obtained when $d=n-1$ by the following theorem.

THEOREM 3. Let $f(z)=P(z) Q(z)^{-1}$, where $P(z)=z^{n}+a_{n-1} z^{n-1}+\cdots+a_{0}$, $Q(z)=b_{n-1} z^{n-1}+\cdots+b_{0}, b_{n-1} \neq 0$, and the Julia set bounded. Then

$$
\int f^{m+1}(z) f^{m}(z) d u(z)=n^{-1}\left(b_{n-1} M_{2}+a_{n-1} M_{1}\right)
$$

with

$$
\begin{aligned}
& M_{1}=-\left(n-b_{n-1}\right)^{-1} a_{n-1}, \\
& M_{2}=\left(n-b_{n-1}^{2}\right)^{-1}\left\{s_{2}-a_{n-1}\left(n-b_{n-1}\right)^{-1}\left(b_{n-2}-a_{n-1} b_{n-1}+\left(s_{1}-t_{1}\right) b_{n-1}\right)\right\} .
\end{aligned}
$$

Proof. By the $f$-invariance of $u$ we have

$$
\begin{aligned}
\int f^{m+1}(z) f^{m}(z) d u(z) & =\int f(z) z d u(z) \\
& =n^{-1} \int z \sum_{i=1}^{n} z_{i}^{1}(z) d u(z)=n^{-1} \int z\left(b_{n-1} z-a_{n-1}\right) d u(z) \\
& =n^{-1}\left(b_{n-1} M_{2}-a_{n-1} M_{1}\right),
\end{aligned}
$$

and the theorem follows from (1).

REMARK 1. This theorem gives us necessary and sufficient conditions for $\int f^{m}(z) f^{n}(z) d u(z)=0$ for $m>n$ in terms of the coefficients of $f^{m-n}$, as explained by the next theorem.

THEOREM 4. Let $f(z)$ be a rational map as above such that $a_{n-1}=a_{n-2}=0$, $b_{n-1} \neq n, b_{n-1}^{2} \neq n$. Then $\left\{f^{n}(z)\right\}$ satisfies $\int f^{m}(z) f^{n}(z) d u(z)=0$ for $m \neq n$.

PROoF. Since $s_{1}=-a_{n-1}$ and $s_{2}=a_{n-1}^{2}-2 a_{n-2}$, we have from (1) that $M_{1}=0$ and $M_{2}=0$. For $m>n, f^{m-n}$ and $f$ have the same maximal measure [6]. Therefore, using the same argument as for Theorem 3 ,

$$
\begin{aligned}
\int f^{m}(z) f^{n}(z) d u(z) & =\int f^{m-n}(z) z d u(z)=\int(c z+d) z d u(z) \\
& =c M_{2}+d M_{1}, \quad \text { where } c, d \in \mathbb{C} .
\end{aligned}
$$

Since $M_{1}=M_{2}=0$, the proposition follows.

REMARK 2. If one considers the case of real rational maps such that the Julia set is contained on $\mathbb{R}$, one recovers orthogonality with respect to the usual inner product. Note that $b_{n-1} \neq n$ and $b_{n-1}^{2} \neq n$ are automatically satisfied when $J(f)$ is bounded.

\section{REFERENCES}

1. H. Brolin, Invariant sets under iteration of rational functions, Ark. Mat. 6 (1966), 103-144.

2. M. F. Barnsley, J. S. Geronimo and A. N. Harrington, Orthogonal polynomial associated with invariant measure on the Julia set, Bull. Amer. Math. Soc. (N.S.) 7 (1982), 381-384.

3. M. F. Barnsley and A. N. Harrington, Moments of balanced measures on Julia sets, Trans. Amer. Math. Soc. 284 (1984), 271-280. 
4. D. Bessis, D. Mentha and P. Moussa, Orthogonal polynomials on a family of Cantor sets and the problem of iterations of quadratic mapping, Lett. Math. Phys. 6 (1982), 123-140.

5. D. Bessis and P. Moussa, Orthogonality properties of iterated polynomial mapping, Comm. Math. Phys. 88 (1983), 503-529.

6. A. Freire, A. Lopes and R. Mañé, An invariant measure for rational maps, Bol. Soc. Brasil. Mat. 14 (1983), 45-62.

7. E. Hille, Analytic function theory, Blaisdell, New York, 1963.

8. V. Lubitsh, Entropy properties of rational endomorphisms of the Riemann sphere, Ergodic Theory Dynamical Systems 3 (1983) 351-383.

9. A. Lopes, Equilibrium measures for rational maps, Ergodic Theory Dynamical Systems (to appear).

10. R. Mañé, On the uniqueness of the maximizing measure for rational maps, Bol. Soc. Brasil. Mat. 14 (1983), 27-43.

11. A. Manning, The dimension of the maximal measure for a rational map, Ann. of Math. (2) 3 (1984), 425-430.

12. D. Ruelle, Repellers for real analytic maps, Ergodic Theory Dynamical Systems 2 (1982), 99-107.

Instituto de Matemática, Universidade Federal do Rio Grande do Sul, Av. Bento Gon Çalves 9500, Campus do Vale, 91500 Porto Alegre, RS, Brasil 